\title{
Martel, V. (2018). Développer des compétences de recherche et de littératie au primaire et au secondaire: Former à l'enquête en classe d'histoire. Montréal, QC : Éditions JFD.
}

\section{RECENSION}

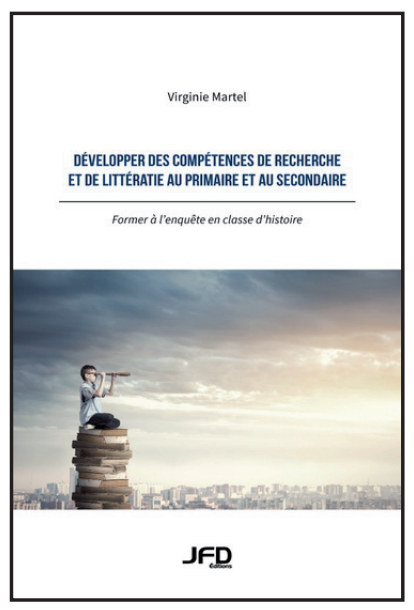

Dans Développer des compétences de recherche et de littératie au primaire et au secondaire: Former à l'enquête en classe d'histoire, la professeure en sciences de l'éducation à l'UQAR Virginie Martel propose des " ponts théoriques et pratiques » entre les didactiques du français et de l'histoire afin d'adapter la méthode historique au contexte scolaire. L'histoire étant essentiellement une discipline d'enquête, elle implique naturellement une grande part de lecture et d'interprétation de plusieurs types de documents, selon une méthodologie à la fois précise et polyvalente. Si l'on souhaite amener l'élève à " problématiser le passé » comme le fait l'historien, il est essentiel de structurer sa démarche d'investigation et de lui présenter les outils conceptuels propres à la discipline historique. Or, ces outils font appel à des compétences de littératie relativement complexes, que l'enseignant d'histoire ne doit pas prendre comme des acquis chez l'élève. Pour l'auteure, le cours d'histoire devrait donc privilégier un modelage de la pensée historienne en développant activement chez l'élève des compétences d'acquisition de l'information (lecture) et de communication de ses interprétations (écriture). On le constate : cette démarche pédagogique appelle naturellement au croisement avec la didactique du français.

Martel souligne d'ailleurs l'importance grandissante de la «littératie multimodale" en classe d'histoire, alors que l'essor technologique du XXI siècle a révolutionné l'accès à l'information, qui s'est elle-même multipliée et diversifiée dans des proportions qui demeuraient inimaginables il y a à peine trois décennies. Parallèlement, l'histoire scolaire s'est considérablement déliée de son approche traditionnelle, qui a longtemps maintenu 
l'élève dans la passivité en privilégiant la transmission de faits établis. La discipline a ainsi cherché à se renouveler en n'exigeant plus de l'élève la mémorisation de récits préconstruits, mais en le plaçant plutôt dans une posture d'enquête et de problématisation des réalités historiques afin qu'il interroge et interprète lui-même le passé et ses traces. Pour y parvenir, le croisement disciplinaire suggéré par Martel oriente les stratégies de lecture propres à la didactique du français scolaire vers le décodage méthodique des documents historiques en classe. Ces stratégies sont en effet complémentaires à la méthodologie de l'enquête en histoire, car elles facilitent l'analyse systématique et rationalisée des documents, quelles que soient leur forme, leur intention ou leur fiabilité. Comme le souligne l'auteure, tout type de document historique, qu'il provienne du monde scientifique ou profane, exprime une réalité historique interprétable en classe. Dans cette optique, l'approche de Martel est intéressante, car elle place le développement des compétences de décodage et de communication en amont de la discipline historique, ce qui rend cette dernière beaucoup plus prompte à être mise à profit par l'élève dans différents contextes scolaires ou sociaux pouvant faire appel au sens critique, tel que le traitement de l'actualité ou des sollicitations politiques.

Structurer la démarche d'analyse documentaire et de communication des interprétations historiques chez l'élève n'est certes pas une tâche facile, mais le croisement didactique avec le français que présente l'auteure a cet avantage de s'appuyer sur des compétences développées aussi en dehors du cours d'histoire, et de surcroît dans une matière qui occupe une place de première importance dans le parcours scolaire des élèves. Le prolongement de ces compétences du français vers l'histoire s'effectue toujours en regard des heuristiques et des approches didactiques de la discipline historique, dont Martel présente une synthèse rigoureuse et actualisée, qui caractérise bien le renouvellement que connaît l'histoire scolaire au Québec. En ce sens, cet ouvrage sera d'une grande utilité aux étudiants et aux professionnels en éducation qui ne seraient pas familiers avec les pratiques scolaires contemporaines du Québec ou de la francophonie. Louvrage se prête également bien à une consultation sélective et ponctuelle de ses différents chapitres, ce qui en fait un choix pertinent comme livre de référence dans un cours universitaire en éducation. La lecture continue détectera toutefois la répétition fréquente des concepts centraux au livre, au point de l'alourdir considérablement, mais cette répétitivité devient tout à fait appropriée dans un cadre académique où la lecture serait dirigée autour de chapitres sélectionnés, chacun référant abondamment aux fondements théoriques qui le composent. Par ailleurs, l'inclusion de l'éducation primaire dans l'ouvrage peut sembler superflue - tout comme sa mention dans le titre - puisque le sujet n'est que très peu abordé, alors que les dispositifs et intentions pédagogiques qui sont répertoriés semblent plutôt adaptés au niveau secondaire.

Virginie Martel réunit ici une compilation exhaustive des différents outils d'analyse critique de l'histoire scolaire, certains complémentaires et d'autres concurrents, laissant le soin au lecteur de les comparer et de s'approprier les dispositifs de son choix. Pour l'enseignant d'histoire, il s'agit d'un ouvrage d'autant plus intéressant qu'il répertorie des approches didactiques complètes et des activités adaptées à chaque facette de la lecture multimodale, du document informatif textuel, audiovisuel ou numérique jusqu'à l'œuvre de fiction littéraire, cinématographique ou vidéoludique. Aussi, une section sur "l'enquête culturelle » présente au lecteur une démarche originale qui permet d'appliquer la lecture multimodale à l'analyse d'un corpus documentaire selon des thématiques choisies. Les quelque 20 tableaux de synthèse présentant les différentes démarches et stratégies d'enquête sont quant à eux des outils de référence d'une utilité certaine, tant pour l'étudiant en éducation que pour l'enseignant soucieux de sa 
démarche méthodologique au fil de sa planification. Ces divers outils inciteront le lecteur à réfléchir sur ses propres intentions pédagogiques en classe d'histoire, et lui permettront d'envisager concrètement le passage à un enseignement fondé sur l'apprentissage-recherche et la démarche d'enquête.

\section{Pour citer cet article}

Melançon, M. (2019). Martel, V. (2018). Développer des compétences de recherche et de littératie au primaire et au secondaire: Former à l'enquête en classe d'histoire. Montréal, QC : Éditions JFD. Formation et profession. 27(3), 153-155 http://dx.doi.org/10.18162/fp.2019.a181 